\title{
Attosecond-Recollision-Controlled Selective Fragmentation of Polyatomic Molecules
}

\author{
Xinhua Xie, ${ }^{1}$ Katharina Doblhoff-Dier, ${ }^{2}$ Stefan Roither, ${ }^{1}$ Markus S. Schöffler, ${ }^{1}$ Daniil Kartashov, ${ }^{1}$ Huailiang Xu, ${ }^{1,3}$ \\ Tim Rathje, ${ }^{4,5}$ Gerhard G. Paulus, ${ }^{4,5}$ Andrius Baltuška, ${ }^{1}$ Stefanie Gräfe, ${ }^{2}$ and Markus Kitzler ${ }^{1, *}$ \\ ${ }^{1}$ Photonics Institute, Vienna University of Technology, A-1040 Vienna, Austria \\ ${ }^{2}$ Institute for Theoretical Physics, Vienna University of Technology, A-1040 Vienna, Austria \\ ${ }^{3}$ State Key Laboratory on Integrated Optoelectronics, College of Electronic Science and Engineering, \\ Jilin University, Changchun 130012, China \\ ${ }^{4}$ Institute of Optics and Quantum Electronics, Friedrich-Schiller-University Jena, D-07743 Jena, Germany \\ ${ }^{5}$ Helmholtz Institute Jena, D-07743 Jena, Germany \\ (Received 22 August 2012; published 10 December 2012)
}

\begin{abstract}
Control over various fragmentation reactions of a series of polyatomic molecules (acetylene, ethylene, 1,3-butadiene) by the optical waveform of intense few-cycle laser pulses is demonstrated experimentally. We show both experimentally and theoretically that the responsible mechanism is inelastic ionization from inner-valence molecular orbitals by recolliding electron wave packets, whose recollision energy in few-cycle ionizing laser pulses strongly depends on the optical waveform. Our work demonstrates an efficient and selective way of predetermining fragmentation and isomerization reactions in polyatomic molecules on subfemtosecond time scales.
\end{abstract}

DOI: 10.1103/PhysRevLett.109.243001

PACS numbers: $33.80 . \mathrm{Rv}, 42.50 . \mathrm{Hz}, 82.50 . \mathrm{Nd}$

Photodissociation of molecules by femtosecond light pulses is a fascinating phenomenon in terms of both the underlying physics and practical implications to selective control of fragmentation pathway(s) through the shape of the optical waveform. Triggered by optical field ionization, the outcome of the complex electronic and subsequent nuclear dynamics leading to fragmentation is to a large extent predetermined on a subcycle time scale because of a sharp temporal localization of electron release to an attosecond interval at the peak of an intense optical half-cycle. Consequently, as has been shown in the case of diatomic molecules- $\mathrm{D}_{2}$ [1-3], $\mathrm{H}_{2}$ [4,5], $\mathrm{HD}$ [2], $\mathrm{DCl}$ [6] and $\mathrm{CO}$ $[7,8]$ - dissociative ionization strongly depends on the carrier-envelope phase (CEP) of a few-cycle ionizing laser pulse. The CEP dependence in the case of the lightest molecules, $\mathrm{D}_{2} / \mathrm{H}_{2}$, has been linked to a field-driven population transfer between the binding ground state and the dissociative excited state.

In this Letter, we present the first, to our knowledge, CEP control of fragmentation pathways of polyatomic molecules (acetylene, ethylene, 1,3-butadiene). Given the increased number of participating nuclei and a vastly more complex valence electron dynamics and the structure of energy surfaces, it is counterintuitive to expect the CEP dependence of fragmentation pathways to survive in polyatomic molecules. Yet the experimental evidence provided in this Letter reveals a prominent role of the CEP for all three polyatomic species under examination and necessitates a search for a suitably controllable attosecond physical mechanism that persists despite the increased complexity of the system. We argue both experimentally and theoretically, that such a universal attosecond mechanism of quasi-single-cycle fragmentation of large molecules is the field control of the tunneled-out electronic wave packet that is steered by the optical waveform before its recollision with the parent ion. This scenario is indeed reminiscent of the role a light-field-accelerated electron plays in the process of high-harmonic generation [9]. The universality of the CEP effect in polyatomic molecular fragmentation is based on the fact that it is comparatively easy to "encode" the detached electron wave packet with a necessary recollision momentum. Conversely, it is difficult to encode many electronic degrees of freedom in a complex molecule directly with the electric field of the femtosecond pulse.

In our experiments, we focus few-cycle laser pulses with sub-5 fs duration, linearly polarized along the $z$ direction of our lab coordinate system, in an ultrahigh vacuum chamber onto a supersonic molecular gas jet, propagating along $x$, of randomly aligned acetylene $\left(\mathrm{C}_{2} \mathrm{H}_{2}\right)$, ethylene $\left(\mathrm{C}_{2} \mathrm{H}_{4}\right)$, or 1,3-butadiene $\left(\mathrm{C}_{4} \mathrm{H}_{6}\right)$ molecules. Details of the experiment can be found in the Supplemental Material [10]. In short, the three-dimensional momentum vectors of fragment ions created by laser-induced fragmentation of a single molecule were measured by cold target recoil ion momentum spectroscopy $[11,12]$. The duration and the CEP of each few-cycle laser pulse was measured on a single-shot basis by stereodetection of photoelectron spectra in a separate apparatus [13-15], and linked to the momenta of the ionic fragments in the offline data analysis. The peak intensity of the laser pulses on target was determined from separate calibration measurements using single ionization of argon atoms in circularly polarized light [16].

The main experimental results are summarized in Fig. 1. Panels (a)-(c) show the ion yields of different two-body fragmentation channels from the doubly charged ions of 

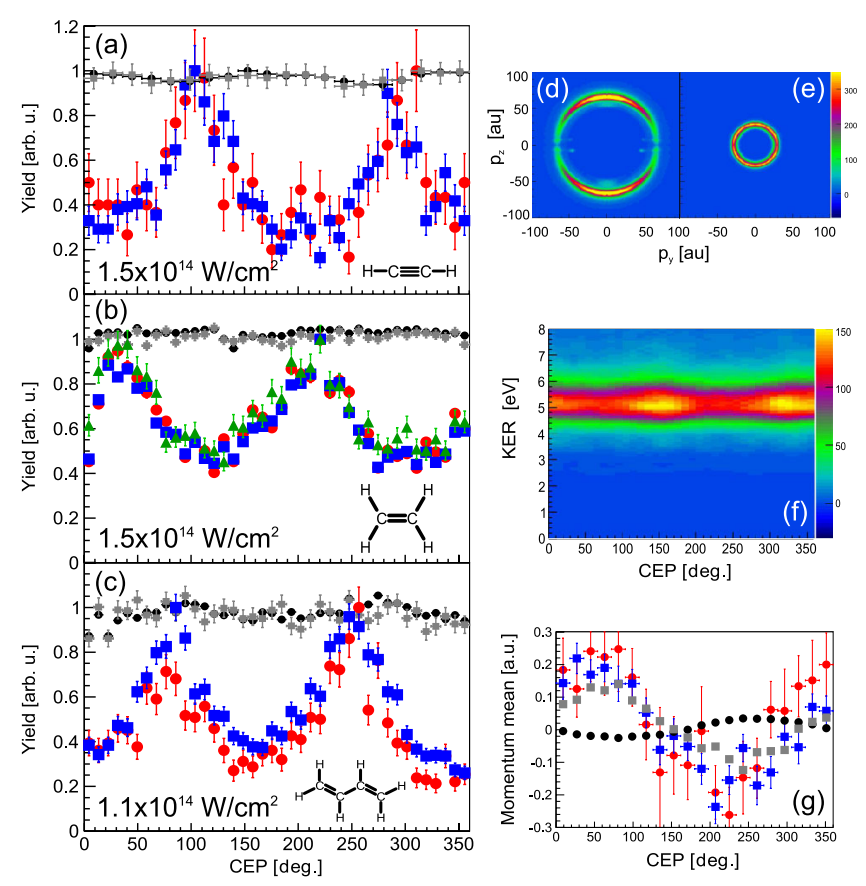

FIG. 1 (color online). (a)-(c) Measured fragmentation and ionization yields, normalized to 1 at their respective maxima, as a function of CEP for different fragmentation channels of acetylene (a), ethylene (b), and 1,3-butadiene (c), measured at the laser intensities indicated in the panels. The various fragmentation reactions are $\mathrm{C}_{2} \mathrm{H}_{2}^{2+} \rightarrow \mathrm{CH}^{+}+\mathrm{CH}^{+}$(red dots) and $\mathrm{C}_{2} \mathrm{H}_{2}^{2+} \rightarrow \mathrm{H}^{+}+\mathrm{C}_{2} \mathrm{H}^{+}$(blue squares) for acetylene (a); $\mathrm{C}_{2} \mathrm{H}_{4}^{2+} \rightarrow \mathrm{CH}_{2}^{+}+\mathrm{CH}_{2}^{+} \quad$ (red dots), $\mathrm{C}_{2} \mathrm{H}_{4}^{2+} \rightarrow \mathrm{H}^{+}+\mathrm{C}_{2} \mathrm{H}_{3}^{+}$ (blue squares), and $\mathrm{C}_{2} \mathrm{H}_{4}^{2+} \rightarrow \mathrm{C}_{2} \mathrm{H}_{2}^{+}+\mathrm{H}_{2}^{+}$(green triangles) for ethylene (b); $\mathrm{C}_{4} \mathrm{H}_{6}^{2+} \rightarrow \mathrm{C}_{2} \mathrm{H}_{3}^{+}+\mathrm{C}_{2} \mathrm{H}_{3}^{+}$(red dots) and $\mathrm{C}_{4} \mathrm{H}_{6}^{2+} \rightarrow$ $\mathrm{CH}_{3}^{+}+\mathrm{C}_{3} \mathrm{H}_{3}^{+}$(blue squares) for 1,3-butadiene (c). The ionization yields of the singly and doubly charged molecular ions are denoted by black dots and gray squares, respectively. (d), (e) Cuts through the measured three-dimensional momentum spectra of $\mathrm{CH}_{2}^{+}$(d) and $\mathrm{H}_{2}^{+}$(e) associated with two of the three fragmentation channels identified for ethylene shown in (b), in a plane along $\left(p_{z}\right)$ and perpendicular $\left(p_{y}\right)$ the laser polarization direction, for $\left|p_{x}\right|<10$ a.u. and integrated over all CEP values. (f) Kinetic energy release (KER) spectrum of the fragmentation reaction $\mathrm{C}_{2} \mathrm{H}_{4}^{2+} \rightarrow \mathrm{CH}_{2}^{+}+\mathrm{CH}_{2}^{+}$as a function of CEP. (g) Mean $p_{z}$ value (integrated over $p_{x}$ and $p_{y}$ ) of the singly (black dots) and doubly (gray squares) charged molecular ions of acetylene as a function of CEP, in comparison with the $p_{z}$ sum of the two fragments from the two fragmentation channels shown in the same color and point style as in (a).

acetylene, ethylene, and 1,3-butadiene as a function of the $\mathrm{CEP}, \varphi_{\mathrm{CE}}$ (which exhibits a phase offset with respect to the absolute CEP, $\varphi$; see Supplemental Material [10]). The yields of all identified fragmentation channels in Fig. 1 exhibit an extraordinarily strong dependence on the CEP with the strongest modulation observed for acetylene (close to $80 \%$ ). The fragmentation yields in Figs. 1(a)-1(c) are the integrals over the three-dimensional momentum spectra of a certain fragmentation channel, uniquely identified by momentum conservation (see Supplemental Material [10]). Figures 1(d) and 1(e) show examples of momentum spectra for ethylene, integrated over all CEP values. The mean momenta of the fragments show only a slight dependence on the CEP. The yields of the singly and doubly charged molecular ions, shown in Figs. 1(a)-1(c), are also widely independent of the CEP. The fragmentation yield, in contrast, is strongly modulated by the CEP, as can be seen from the kinetic energy release spectra, exemplarily shown for the fragmentation channel $\mathrm{C}_{2} \mathrm{H}_{4}^{2+} \rightarrow \mathrm{CH}_{2}^{+}+\mathrm{CH}_{2}^{+}$in Fig. 1(f).

Which light-field dependent mechanism can lead to such a strong CEP dependence of the fragmentation yield? We will discuss this question for the example of acetylene, for which the quantum chemically calculated energy level diagram for the C-C stretch mode is shown in Fig. 2(a). Details about the quantum chemical methods for calculating the energy levels are given in the Supplemental Material [10]. The energy level diagram shows that the dicationic ground state is bound. The observed fragmentation reactions, thus, have to take place on one (or more) excited states. The first dissociative states lie about 5-6 eV above the dicationic ground state and can lead to both fragmentation channels observed for acetylene. Hence, to observe the strong modulation of the fragmentation yield shown in Fig. 1, the population of the involved excited state(s) has to be accomplished by a strongly CEP dependent mechanism.

As the probability for field ionization depends exponentially on the field strength and, therewith, is sensitive to the CEP of few-cycle pulses, simple sequential double ionization could be a candidate for this mechanism. Indeed, the fragmentation channels observed here have also been observed in other experiments with similarly low laser pulse intensities, but longer pulse durations ( $\approx 25-50 \mathrm{fs}$ ), e.g., for 1,3-butadiene [17]. In the present experiments, owing to the short duration and low intensity of the laser pulses, and supported by previous measurements with longer pulses [18], we expect double ionization to occur predominantly via electron recollision. Indeed, Fig. 1 (g) shows that (i) the measured momentum of $\mathrm{C}_{2} \mathrm{H}_{2}^{2+}$ along the laser polarization direction is much larger than the one of $\mathrm{C}_{2} \mathrm{H}_{2}^{+}$, and that (ii) the two momenta point to opposite directions for almost all CEP values. Such CEP dependence has been shown to be caused by double ionization via electron recollision [19]. Furthermore, as the sum momentum of the two emitted fragments shows the same dependence on the CEP as $\mathrm{C}_{2} \mathrm{H}_{2}^{2+}$ [see Fig. 1(g)], the fragmentation reactions are also initiated by recollision ionization (RI). We thus conclude that the observed CEP dependence of the fragmentation yields is most likely due to a strong CEP dependence of RI into the first dissociative states.

In order to elucidate this point we plot in Fig. 2(b) the electron recollision energy in the few-cycle pulses used in 


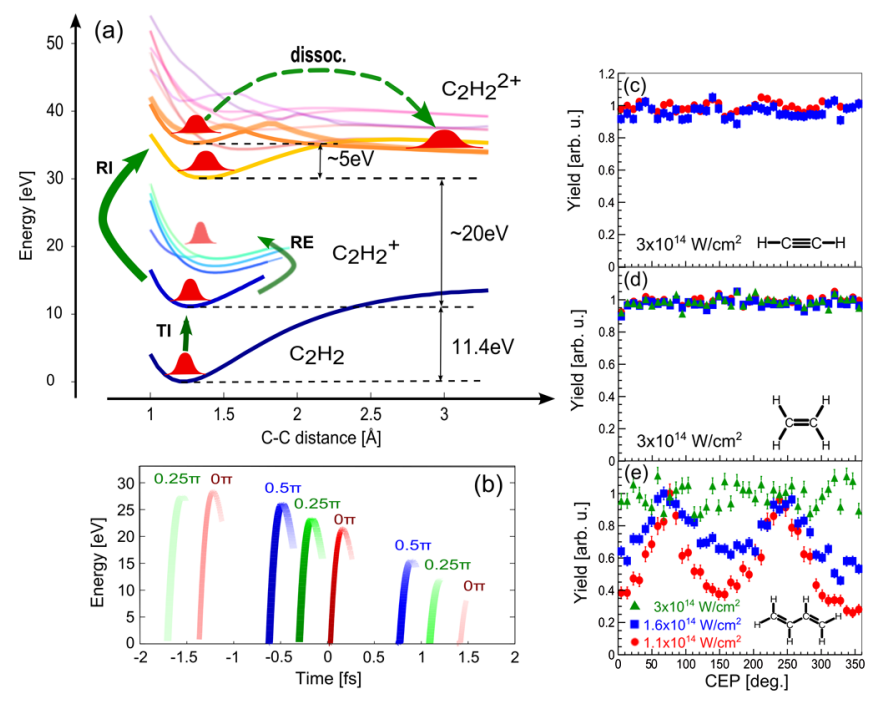

FIG. 2 (color online). (a) Electronic energy levels of the neutral, cation, and dication of acetylene as a function of C-C distance, calculated by $a b$ initio quantum-chemical methods as described in the Supplemental Material [10]. TI, RI, and RE denote tunnel ionization, recollision ionization, and recollision excitation, respectively. (b) Electron recollision energy over the time of ionization calculated for a 4.5 fs (FWHM) long laser pulse with an intensity of $1.5 \times 10^{14} \mathrm{~W} / \mathrm{cm}^{2}$ for the three indicated values of the CEP: 0 (red lines), $0.25 \pi$ (green lines), and $0.5 \pi$ (blue lines). The contribution of each recollision event as determined by the ionization probability is indicated by the color intensity. (c), (d) Fragmentation yields over the CEP of the same channels as in Figs. 1(a) and 1(b) (same color and point styles apply), but measured for a slightly higher intensity (as indicated). (e) Measured intensity dependence of the yield of the fragmentation channel $\mathrm{C}_{4} \mathrm{H}_{6}^{2+} \rightarrow \mathrm{CH}_{3}^{+}+\mathrm{C}_{3} \mathrm{H}_{3}^{+}$over CEP. The intensities are indicated in the figure.

our experiments on acetylene, for different CEP values as a function of birth time, calculated using the simple man's model [20,21]. For this intensity $\left(1.5 \times 10^{14} \mathrm{~W} / \mathrm{cm}^{2}\right)$, the strongly CEP-dependent maximum recollision energy of the dominant recollision event is only $22 \mathrm{eV}$ for $\varphi_{\mathrm{CE}}=0$, while for $\varphi_{\mathrm{CE}}=\pi / 2$ roughly $26 \mathrm{eV}$ are reached. About $20 \mathrm{eV}$ are necessary to reach the ground state of the acetylene dication, see Fig. 2(a). Additional $\sim 5-6 \mathrm{eV}$ are necessary to reach the first dissociative states that lead to the experimentally observed correlated fragments $\mathrm{CH}^{+} / \mathrm{CH}^{+}$and $\mathrm{C}_{2} \mathrm{H}^{+} / \mathrm{H}^{+}$. Thus, the probability for reaching the dissociative state is highest for CEP values around $\pi / 2$, and small for CE phases around 0 . We therefore propose the following scenario [see Fig. 2(a)] for explaining the pronounced fragmentation yield modulations observed in the experiment: An electronic wave packet set free by field ionization dominantly from the highest occupied molecular orbital (HOMO) of the neutral molecule, is driven back by the laser field and doubly ionizes the molecule by electron impact ionization. For CEP values that result in low recollision energy, the second electron will be dominantly removed from the HOMO, leading to ionization to the nondissociative dication's ground state. For CEP values resulting in high recollision energy, the electron can also be removed from a lower lying orbital, which puts the molecular ion into a dissociative excited electronic state. For example, the lowest excited state $\left({ }^{3} \Pi_{u}\right)$ associated with the fragmentation products $\mathrm{C}_{2} \mathrm{H}^{+} / \mathrm{H}^{+}$that we discussed above, is reached by removal of an electron from the HOMO-2 orbital. Because the fragmentation reactions that we investigated in the present experiments take place on electronically excited state surfaces, whose populations are collectively controlled by the CEP, we observe the same CEP dependence for each fragmentation channel of a certain molecule (Fig. 1).

We can test the proposed scenario experimentally simply by increasing the laser peak intensity $I$, since the recollision energy scales linearly with intensity as $E_{\text {rec }} \propto I / 4 \omega^{2}[20,21]$. If the recollision energy is high enough for all CEP values to reach the first dissociative states, the fragmentation yield is expected to be independent of the CEP. Figures 2(c) and 2(d) show the measured yield as a function of CEP of the same fragmentation channels as in Figs. 1(a) and 1(b), but with a twice higher laser intensity leading to twice the maximum recollision energy. As expected, the fragmentation yields now show no discernible modulation with the CEP. Thus, by precise adjustment of the laser intensity, such that the energy threshold to the dissociative states is overcome only for certain CEP values, light-field control of the fragmentation of polyatomic molecules becomes possible. This is demonstrated in Fig. 2(e), for the example of the fragmentation channel $\mathrm{C}_{4} \mathrm{H}_{6}^{2+} \rightarrow \mathrm{CH}_{3}^{+}+\mathrm{C}_{3} \mathrm{H}_{3}^{+}$, where the laser intensity has been fine-tuned in three steps over the dissociation energy threshold, resulting first in a decreased modulation depth of the fragmentation yield, and for even higher intensity in its complete disappearance.

To obtain detailed insight into the light-determined fragmentation dynamics, we now turn to simulations (detailed in the Supplemental Material [10]), performed exemplarily for acetylene, $\mathrm{C}_{2} \mathrm{H}_{2}^{2+}$. Briefly, we use a onedimensional semiclassical model to simulate recollision ionization into the excited dissociative state. The resulting fragmentation probability as a function of CEP and laser intensity is shown in Fig. 3(a). At very low laser intensities below roughly $1.2 \times 10^{14} \mathrm{~W} / \mathrm{cm}^{2}$, the recollision energy of the electron is too small to reach the dissociative state, independent of the CEP. For slightly higher intensities, lower lying electrons can be ionized by RI for certain values of the CEP. With increasing intensity the CEP values, where maximum fragmentation probability is reached, shift. At the same time, the modulation depth decreases [see Fig. 3(b)], since for higher laser intensity and therewith higher electron recollision energy also recollisions for other CEP values have enough energy to ionize a low lying electron. Taking an average over 


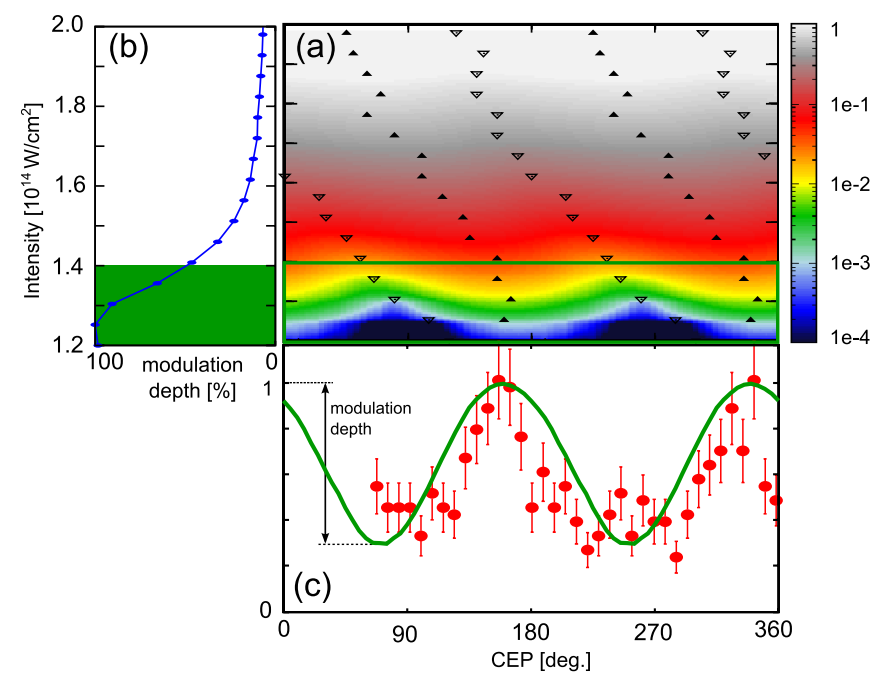

FIG. 3 (color online). Comparison of simulated and measured fragmentation probability of the channel $\mathrm{C}_{2} \mathrm{H}_{2}^{2+} \rightarrow$ $\mathrm{CH}^{+}+\mathrm{CH}^{+}$. (a) Simulated fragmentation probability encoded in color scale as a function of CEP and laser intensity, normalized to its maximum value and calculated as described in the Supplemental Material [10]. The CEP and intensity dependent position of maximum and minimum fragmentation yield is indicated by black upwards and gray downwards pointing triangles, respectively. (b) Modulation depth of the fragmentation probability [as defined in (c)], as a function of laser intensity. (c) Simulated fragmentation probability over CEP (full green line) obtained by averaging of the data in (a) over the intensity range $1.2-1.4 \times 10^{14} \mathrm{~W} / \mathrm{cm}^{2}$ to account for the experimental intensity beam profile [as indicated in (a) and (b)], in comparison with the experimental data from Fig. 1(a) shifted by $60^{\circ}$.

$1.2-1.4 \times 10^{14} \mathrm{~W} / \mathrm{cm}^{2}$, thereby simulating the different intensities within the experimental laser beam profile up to an intensity very close to the experimentally determined value, we obtain the modulation shown in Fig. 3(c), which agrees almost perfectly with the experimental data shifted by $\sim 60^{\circ}$. The CEP shift is attributed to the influence of the long-range Coulomb field on the CEP-dependent energy of electrons from single ionization [22,23], which we have used in our experiment to arbitrarily calibrate the CEP, $\varphi_{\mathrm{CE}}$, and which is also not correctly reproduced in the onedimensional semiclassical model.

The numerical data shown in Fig. 3 have been calculated by neglecting field-driven population transfer between the binding ground state and the dissociative excited states, which in previous work on the dissociative ionization of $\mathrm{D}_{2} / \mathrm{H}_{2}[1-3,24]$ has been shown to be essential in explaining the CEP-dependent charge localization observed in the experiments. Here, we can exclude a noticeable contribution of such a mechanism to the experimentally observed CEP dependence of the fragmentation yield shown in Fig. 1, as it would be incompatible with the observation that for each of the molecules all fragmentation channels show the same CEP dependence. Fielddriven population dynamics, however, would happen on considerably different time scales for breakage of the C-C and $\mathrm{H}-\mathrm{C}$ bonds, respectively, due to the large difference in the fragments' reduced masses $\left(\mathrm{m}_{\mathrm{CC}} / \mathrm{m}_{\mathrm{HC}} \approx 6.8\right)$. In order to confirm this statement, we have performed reduceddimensional quantum dynamical calculations by solving the time-dependent nuclear Schrödinger equation for the four strongest dipole-coupled electronic states of acetylene subjected to the experimental laser parameters (see Supplemental Material [10] for details). The contribution of the field-driven population transfer to the CEP-dependent modulation of the fragmentation yield due to recollision-induced ionization was found to be very small $(<1 \%)$.

Electron recollision, however, can not only promote the second electron directly into the continuum, but can also excite the molecular cation, which may then be further ionized by the laser field [25-27]. We anticipate that this mechanism, dubbed recollision-induced excitation (RE) and subsequent field ionization (RESI), can, in principle, also be used for CEP control of polyatomic molecular fragmentation with few-cycle pulses even at peak intensities that prohibit direct population of the first excited dicationic state. In this case, however, as many of the closely spaced higher excited states in the cation can be populated by RE, the CEP selectivity becomes dominantly dependent on the field-sensitive ionization rate during the field ionization step. As a result, the yields of different fragmentation channels as well as the yield of the dication are likely to show different CEP-modulation depths and possibly different CEP dependence. This is not observed in our experiments. Furthermore, we have estimated that at the intensity of our experiments and the channels observed, RESI is considerably less probable than direct RI to the first dissociative excited state. We therefore conclude that the contributions to the observed CEP modulation of the fragmentation yield due to RESI, while probably important for lower intensities, are insignificant for the here demonstrated higher intensity case that permits direct RI from lower lying orbitals.

In conclusion we have experimentally demonstrated for the first time control over various fragmentation reactions of polyatomic molecules using intense few-cycle laser pulses. Fragmentation reactions of polyatomic molecules are essential building blocks of chemistry. Equally important, however, are preceding isomerization reactions. In our experiment we observe, for example, two channels that involve molecular restructuring prior to the breakage of a molecular bond: formation of $\mathrm{H}_{2}^{+}$for ethylene and a proton migration reaction for 1,3-butadiene [see Fig. 1]. These results demonstrate that RI with intensity-tuned few-cycle laser pulses can be used as a very efficient tool not only to initiate (or suppress) fragmentation but also isomerization reactions in polyatomic molecules with high sensitivity. Our work, thus, demonstrates an efficient and selective, yet straightforward way of predetermining fragmentation and 
isomerization reactions in polyatomic molecules on subfemtosecond time scales.

This work was partly financed by the Austrian Science Fund (FWF), Grants No. P21463-N22, No. V193-N16, and No. I274-N16, by a SIRG grant from the ERC, and by a grant from the National Natural Science Foundation of China (No. 11074098).

*Corresponding author. markus.kitzler@tuwien.ac.at

[1] M. F. Kling, C. Siedschlag, A. J. Verhoef, J. I. Khan, M. Schultze, T. Uphues, Y. Ni, M. Uiberacker, M. Drescher, F. Krausz, and M. J. J. Vrakking, Science 312, 246 (2006).

[2] M. Kling, C. Siedschlag, I. Znakovskaya, A. Verhoef, S. Zherebtsov, F. Krausz, M. Lezius, and M. Vrakking, Mol. Phys. 106, 455 (2008).

[3] I. Znakovskaya, P. von den Hoff, G. Marcus, S. Zherebtsov, B. Bergues, X. Gu, Y. Deng, M. Vrakking, R. Kienberger, F. Krausz, R. de Vivie-Riedle, and M. Kling, Phys. Rev. Lett. 108, 063002 (2012).

[4] M. Kremer, B. Fischer, B. Feuerstein, V. L. B. de Jesus, V. Sharma, C. Hofrichter, A. Rudenko, U. Thumm, C. D. Schröter, R. Moshammer, and J. Ullrich, Phys. Rev. Lett. 103, 213003 (2009).

[5] B. Fischer, M. Kremer, T. Pfeifer, B. Feuerstein, V. Sharma, U. Thumm, C. Schröter, R. Moshammer, and J. Ullrich, Phys. Rev. Lett. 105, 223001 (2010).

[6] I. Znakovskaya, P. von den Hoff, N. Schirmel, G. Urbasch, S. Zherebtsov, B. Bergues, R. de Vivie-Riedle, K.-M. Weitzel, and M. F. Kling, Phys. Chem. Chem. Phys. 13, 8653 (2011).

[7] I. Znakovskaya, P. von Den Hoff, S. Zherebtsov, A. Wirth, O. Herrwerth, M. Vrakking, R. de Vivie-Riedle, and M. Kling, Phys. Rev. Lett. 103, 103002 (2009).

[8] Y. Liu, X. Liu, Y. Deng, C. Wu, H. Jiang, and Q. Gong, Phys. Rev. Lett. 106, 073004 (2011).

[9] P. Corkum, Phys. Rev. Lett. 71, 1994 (1993).

[10] See Supplemental Material at http://link.aps.org/ supplemental/10.1103/PhysRevLett.109.243001 for details of the experiments and simulations.

[11] R. Dörner, V. Mergel, O. Jagutzki, L. Spielberger, J. Ullrich, R. Moshammer, and H. Schmidt-Böcking, Phys. Rep. 330, 95 (2000).
[12] L. Zhang, S. Roither, X. Xie, D. Kartashov, M. Schöffler, H. Xu, A. Iwasaki, S. Gräfe, T. Okino, K. Yamanouchi, A. Baltuska, and M. Kitzler, J. Phys. B 45, 085603 (2012).

[13] T. Wittmann, B. Horvath, W. Helml, M. Schätzel, X. Gu, A. Cavalieri, G. Paulus, and R. Kienberger, Nat. Phys. 5, 357 (2009).

[14] A. M. Sayler, T. Rathje, W. Müller, C. Kürbis, K. Rühle, G. Stibenz, and G. G. Paulus, Opt. Express 19, 4464 (2011).

[15] A. M. Sayler, T. Rathje, W. Müller, K. Rühle, R. Kienberger, and G. G. Paulus, Opt. Lett. 36, 1 (2011).

[16] C. Maharjan, A. Alnaser, X. Tong, B. Ulrich, P. Ranitovic, S. Ghimire, Z. Chang, I. Litvinyuk, and C. Cocke, Phys. Rev. A 72, 041403 (2005).

[17] H. Xu, T. Okino, K. Nakai, K. Yamanouchi, S. Roither, X. Xie, D. Kartashov, M. Schöffler, A. Baltuska, and M. Kitzler, Chem. Phys. Lett. 484, 119 (2010).

[18] C. Cornaggia and P. Hering, Phys. Rev. A 62, 023403 (2000).

[19] B. Bergues, M. Kübel, N. G. Johnson, B. Fischer, N. Camus, K. J. Betsch, O. Herrwerth, A. Senftleben, A. M. Sayler, T. Rathje, T. Pfeifer, I. Ben-Itzhak, R. R. Jones, G. G. Paulus, F. Krausz, R. Moshammer, J. Ullrich, and M. F. Kling, Nature Commun. 3, 813 (2012).

[20] G. G. Paulus, W. Becker, W. Nicklich, and H. Walther, J. Phys. B 27, L703 (1994).

[21] M. Lewenstein, K. Kulander, K. Schafer, and P. Bucksbaum, Phys. Rev. A 51, 1495 (1995).

[22] S. Chelkowski, A. Bandrauk, and A. Apolonski, Phys. Rev. A 70, 013815 (2004).

[23] S. Chelkowski and A.D. Bandrauk, Phys. Rev. A 71, 053815 (2005).

[24] S. Gräfe and M. Y. Ivanov, Phys. Rev. Lett. 99, 163603 (2007).

[25] R. Kopold, W. Becker, H. Rottke, and W. Sandner, Phys. Rev. Lett. 85, 3781 (2000).

[26] B. Feuerstein, R. Moshammer, D. Fischer, A. Dorn, C. Schröter, J. Deipenwisch, J. C. Lopez-Urrutia, C. Höhr, P. Neumayer, J. Ullrich, H. Rottke, C. Trump, M. Wittmann, G. Korn, and W. Sandner, Phys. Rev. Lett. 87, 043003 (2001).

[27] A. Rudenko, K. Zrost, B. Feuerstein, V. de Jesus, C. Schröter, R. Moshammer, and J. Ullrich, Phys. Rev. Lett. 93, 253001 (2004). 